\title{
PA-137 ASSESSMENT OF THE ENDEMICITY STATUS OF SCHISTOSOMIASIS AND SOIL-TRANSMITTED HELMINTHIASIS IN THE GAMBIA
}

Yaya Camara, Bakary Sanneh. Ministry of Health and Social Welfare, The Gambia

10.1136/bmjgh-2016-000260.163

Background The Ministry of Health and Social Welfare, The Gambia with support from WHO and Task Force for Global Health (TFGH), conducted a national endemicity mapping survey for schistosomiasis $(\mathrm{SCH})$ and soiltransmitted helminths (STH) to establish their endemicity status. The survey was meant to provide baseline information on endemicity in order to plan and implement strategic interventions. This is a critical step towards NTD elimination by 2020 .

Methods A cross-section of fifty school-aged children (SAC, 25 boys and 25 girls) per school was sampled in 209 schools countrywide. Eligible SAC of 7 to 14 years old were randomly selected using formula $(\mathrm{n} / 50)$ where $\mathrm{n}=$ total eligible pupils per school. Stool, urine and finger prick samples provided, were examined for SCH and STH using Kato-Katz, urine filtration, dip-stick and CCA techniques.

Results National prevalence of schistosomiasis and soiltransmitted helminthiasis were $4.3 \%$ and $2.5 \%$, respectively. At district level, Niani had the highest prevalence of $\mathrm{SCH}$, recording 22\%. Whereas for STH, Banjul, the capital city, had the highest prevalence, recording $55 \%$, followed by $22 \%$ prevalence in Kombo South. Schistosoma haematobium is the most dominant parasitic infection in The Gambia. Fourteen (38\%) districts in the country are co-endemic for both STH and $\mathrm{SCH}$. Generally, male pupils are more infected with urinary schistosomiasis than females.

Conclusions It was established that 19 (45\%) of districts mapped are endemic for schistosomiasis; thus the need for treatment with praziquantel. Twenty (47\%) of districts mapped are endemic for soil-transmitted helminthiasis at varying rates. However, only two STH endemic districts, Banjul (55\%), and Kombo South (22\%), within the high and very high prevalence rates of endemicity, are eligible for treatment with albendazole. 